\title{
Onset of time-dependence in a double-gyre circulation: Barotropic basin modes versus classical baroclinic modes
}

\author{
by Hristina G. Hristova ${ }^{1}$, Henk A. Dijkstra ${ }^{2}$ and Michael A. Spall ${ }^{3}$
}

\begin{abstract}
Using a fully-implicit high-resolution two-layer quasi-geostrophic model combined with pseudoarclength continuation methods, we perform a bifurcation analysis of double-gyre ocean flows to study their initial oscillatory instabilities. In this model, both wind- and thermally-forced flows can be represented. We demonstrate that on the branch of anti-symmetric steady-state solutions the ratio, $\Omega$, of the flow advective speed to the long internal Rossby wave speed determines the type of oscillatory modes to first become unstable. This is the same nondimensional parameter that controls the shape of the geostrophic contours in the linear limit of the circulation. For large values of $\Omega$, the first Hopf bifurcations correspond to the classical baroclinic modes with inter-monthly time periods arising from shear instability of the flow. For small values of $\Omega$, the first Hopf bifurcations correspond instead to barotropic Rossby modes with shorter, monthly periods arising from mixed barotropic-baroclinic instability of the flow. By considering both a wind-forced and a thermally-forced ocean, we show that this is a robust feature that does not depend on the type of forcing driving the circulation.
\end{abstract}

\section{Introduction}

An important problem in physical oceanography is understanding the physics of the timemean ocean circulation and its variability on timescales from several months to decades. Part of this variability is in response to the variable atmospheric forcing, but part is related to the so-called internal ocean variability. Nonlinear systems, such as the ocean circulation, can exhibit complex time-dependent behavior on a variety of timescales even under steady forcing conditions as a result of internal feedbacks between perturbations and mean flow, when certain thresholds for instabilities are exceeded (Dijkstra, 2005).

It has been demonstrated that for the wind-driven double-gyre circulation only a small number of internal modes of variability contribute to its complex transient behavior - the barotropic basin modes, the classical baroclinic modes, the gyre modes and the wall-trapped modes (Nauw et al., 2004). These modes differ by their periods, horizontal and vertical structure, and underlying physical mechanisms. They have been identified as the major

1. Joint Institute for the Study of the Atmosphere and Ocean, University of Washington, Seattle, Washington, 98195, U.S.A.email: hristina@uw.edu

2. Institute for Marine and Atmospheric Research Utrecht, Utrecht University, Utrecht, The Netherlands.

3. Woods Hole Oceanographic Institution, Woods Hole, Massachusetts, 02543, U.S.A. 
players for the mid-latitude ocean circulation variability in an hierarchy of numerical models ranging from simple QG flat-bottom barotropic models (Cessi and Ierley, 1995; Jiang et al., 1995; Dijkstra and Katsman, 1997; Primeau, 2002) to shallow-water and primitive equations models with realistic coastline and bathymetry (Dijkstra and Molemaker, 1999; Schmeits and Dijkstra, 2000).

Amongst these modes, the gyre modes are the only truly low-frequency internal modes of the double-gyre circulation with periods on inter-annual to decadal timescales, depending on the particular parameter choice, and are associated with weakening and strengthening of the mid-latitude jet (Dijkstra and Katsman, 1997; Simonnet and Dijkstra, 2002). The walltrapped modes on the other hand are high-frequency (monthly timescales) oscillations but, as their name suggests, confined to the region of the western boundary and resulting from instabilities of the viscous boundary layer (Cessi and Ierley, 1993). Our focus here is on the remaining two internal modes, the barotropic basin modes and the classical baroclinic modes, which are both oscillations with periods up to inter-monthly and annual timescales. They can be found as the first Hopf bifurcations on the branches of anti-symmetric and asymmetric steady-state solutions for the double-gyre circulation. As such, they influence to a large degree the transient behavior of the system. We will demonstrate that which kind of oscillatory mode appears as the leading instability on the branch of anti-symmetric steady-state solutions, depends on the Rossby deformation radius, or more precisely on the relative size of the long internal Rossby wave basin crossing timescale to the flow advective timescale, a nondimensional parameter that we call $\Omega$ in this paper. Incidentally, this is the same nondimensional parameter that controls the shape of the geostrophic contours, suggesting that there is a relationship between the transient behavior of the system and the presence of closed geostrophic contours in the weakly nonlinear limit.

A new aspect in this study is that we consider the onset of time-dependence in the case of both a wind-driven and a thermally-driven ocean. The thermal forcing is included in the context of a QG model through the introduction of a non-zero cross-isopycnal flux parameterized as relaxation of the interface displacement, which is equivalent to the vertically averaged temperature, toward some externally specified profile (Pedlosky and Spall, 2005). This can be interpreted as a very crude representation of the vertical mixing in the thermocline that leads to water exchange between the deep and upper ocean. While the initial bifurcation diagram for a wind-driven double-gyre flow has been extensively studied, there is no such previous analysis in the case of a thermally-forced flow. Thus, we will present here for the first time the bifurcation diagram for a thermally-driven double-gyre flow. We will also show that the relationship between the initial Hopf bifurcations of the system and the parameter $\Omega$ that controls the shape of the geostrophic contours in the weakly nonlinear limit, holds for both a wind-driven and a thermally-driven double-gyre.

The plan for the presentation is as follows. In Section 2 we will define the model and describe the numerical methods used for the calculations. In Section 3 some initial results about the typical bifurcation diagrams for a wind-driven and a thermally-driven double-gyre are presented. Section 4 concentrates on analyzing the dependence of the type of oscillatory 
a)

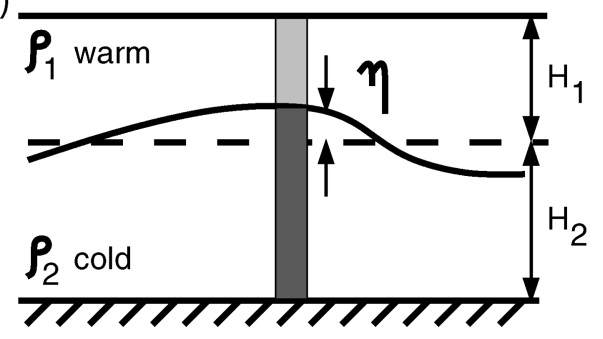

b)

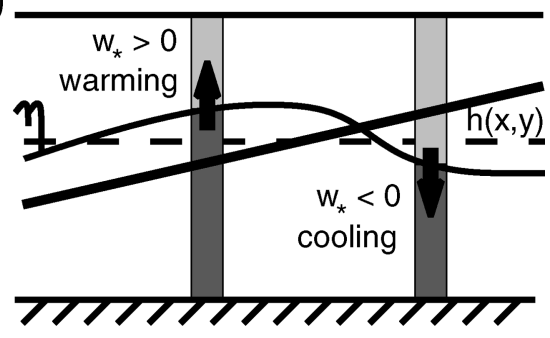

Figure 1. Illustration of the thermal forcing parameterization. a) The upward interface displacement in a 2-layer QG model is representative of the vertically averaged temperature - for example, $\eta$ positive means a cold anomaly. b) The cross-isopycnal velocity $w_{*}$ is parameterized as relaxation of the interface $\eta$ toward an externally specified height $h(x, y)=h_{0} S_{T}(x, y)$ on a timescale $\gamma$.

instabilities on the parameter $\Omega$ with some final conclusions and implications presented in Section 5.

\section{Model formulation and numerical methods}

\section{a. The model}

One of the simplest models that offers insights into the nonlinear dynamics of the doublegyre ocean circulation is the classical quasi-geostrophic (QG) layer model (Pedlosky, 1987). We are using here a 2-layer version of the model, similar to that in Dijkstra and Katsman (1997). The ocean, confined to a flat-bottom square basin of size $L \times L$, is represented by two density layers with thicknesses at rest, $H_{1}$ and $H_{2}$, and reduced gravity $g^{\prime}=g\left(\rho_{2}-\rho_{1}\right) / \rho_{0}$. Dissipation in the form of Laplacian eddy viscosity $\nu$ is applied. The circulation is forced at the surface by a wind-stress curl with magnitude $\left(\tau_{0} / L\right) S_{w}(x, y)$, where $S_{w}(x, y)$ is a nondimensional function describing the spatial variation of the wind forcing. However, unlike most QG models, we have relaxed the assumption of adiabatic motion. Instead, we have included a non-zero cross-isopycnal flux $w_{*}$, parameterized as relaxation of the interface displacement $\eta$ to some prescribed equilibrium profile on a timescale $\gamma$,

$$
w_{*}=\frac{1}{\gamma}\left(\eta-h_{0} S_{T}(x, y)\right) .
$$

In the expression above, $h_{0}$ is the equilibrium profile amplitude in meters, $S_{T}(x, y)$, a nondimensional function describing its spatial variation, while the interface displacement $\eta$ is given in terms of the streamfunctions $\psi_{1}$ and $\psi_{2}$ for the upper and lower layer, respectively, by $\eta=-f_{0}\left(\psi_{1}-\psi_{2}\right) / g^{\prime}$.

Note that the interface displacement in a 2-layer model is proportional to the vertically averaged temperature field anomaly - a positive value for $\eta$ (or an upward displacement) signifies a thinner upper layer and a cold anomaly (Fig. 1). The parameterization for $w_{*}$ is therefore nothing else but a relaxation of the vertically averaged temperature toward some 
externally specified profile. This parameterization is used commonly in atmospheric QG models, where it is referred to as radiative damping (Held, 2000). In the oceanic context, it can be thought of as representing the effect of vertical mixing in the thermocline leading to water properties transformation and water exchange between the upper and deep ocean (Pedlosky and Spall, 2005). In the remainder of this paper, we refer to the cross-isopycnal flux $w_{*}$ as thermal forcing.

Using the basin width $L$, a typical horizontal velocity $U$, and an advective timescale $L / U$, the nondimensional equations describing the ocean circulation become

$$
\begin{aligned}
& \frac{\partial}{\partial t}\left(\nabla^{2} \psi_{1}-F_{1}\left(\psi_{1}-\psi_{2}\right)\right)+J\left(\psi_{1}, \nabla^{2} \psi_{1}-F_{1}\left(\psi_{1}-\psi_{2}\right)\right)+\beta \frac{\partial \psi_{1}}{\partial x} \\
& \quad=U_{w} S_{w}(x, y)-F_{1} w_{*}+\frac{1}{R e} \nabla^{4} \psi_{1}, \\
& \frac{\partial}{\partial t}\left(\nabla^{2} \psi_{2}+F_{2}\left(\psi_{1}-\psi_{2}\right)\right)+J\left(\psi_{2}, \nabla^{2} \psi_{2}+F_{2}\left(\psi_{1}-\psi_{2}\right)\right)+\beta \frac{\partial \psi_{2}}{\partial x} \\
& \quad=F_{2} w_{*}+\frac{1}{R e} \nabla^{4} \psi_{2}, \\
& w_{*}=-\frac{1}{\Omega \delta_{T}}\left(\psi_{1}-\psi_{2}+U_{T} S_{T}(x, y)\right),
\end{aligned}
$$

where $J$ is the Jacobian, defined as $J(a, b)=(\partial a / \partial x)(\partial b / \partial y)-(\partial a / \partial y)(\partial b / \partial x)$.

The following nondimensional parameters appear in the equations: the nondimensional $\beta$ parameter, the Reynolds number $R e$, the ratio $\Omega$ of the advective speed to the long baroclinic Rossby wave speed, the ratio $\delta_{12}$ of the layer depths, a thermal scale $\delta_{T}$ equal to the ratio of the restoring timescale to the time needed for a long Rossby wave to cross the basin and finally two velocity scales, $U_{w}$ and $U_{T}$, defined respectively by the amplitudes of the applied wind and thermal forcing

$$
\begin{aligned}
& \beta=\frac{\beta_{0} L^{2}}{U}, \quad R e=\frac{U L}{\nu}, \quad \Omega=\frac{U}{\beta_{0} R_{d}^{2}}, \quad \delta_{12}=\frac{H_{1}}{H_{2}}, \quad \delta_{T}=\frac{\gamma \beta_{0} R_{d}^{2}}{L}, \\
& U_{T}=\frac{g^{\prime} h_{0}}{U f_{0} L}, \quad U_{w}=\frac{\tau_{0} L}{U^{2} \rho_{0} H_{1}} .
\end{aligned}
$$

In addition, in Eqs. (2a) and (2b) the notations $F_{1}=\left(H_{2} / H\right) \beta \Omega$ and $F_{2}=\left(H_{1} / H\right) \beta \Omega$ have been used for shorter writing, where $H=H_{1}+H_{2}$ is the total depth of the ocean.

For all calculations presented here, the spatial variation of the forcing terms is assumed to be zonally uniform with a sine dependence on latitude, i.e $S_{w}(y)=S_{T}(y)=-\sin (2 \pi y)$. This is meant to represent an idealized subtropical/subpolar gyre circulation with the subtropical gyre being warmed and the subpolar gyre, cooled. The sine forcing implies that there is no net wind-stress curl and no net heating/cooling applied to the system. No-normal flow and no-slip boundary conditions are applied on the basin boundaries, which translates into $\psi_{n}=c_{n}(t)$ and $\nabla \psi_{n} \cdot \hat{\mathbf{n}}=0$ on the walls. The values $c_{n}(t)$ of the streamfunction at 
the walls are determined from the additional condition of mass conservation, which in the presence of cross-isopycnal flux is

$$
\iint \frac{\partial}{\partial t}\left(\psi_{1}-\psi_{2}\right)+\frac{1}{\Omega \delta_{T}}\left(\psi_{1}-\psi_{2}+U_{T} S_{T}(x, y)\right) d x d y=0 .
$$

The mass conservation condition is satisfied accurately for all solutions presented here.

The main goal of this paper is to explore the dependence on $\Omega$ of the onset of timedependent circulation as the Reynolds number is increased. Note that in QG models, it is usually the Froude number $F=\beta \Omega=L^{2} / R_{d}^{2}$ that is introduced instead of $\Omega$. However, we show that the ratio $\Omega$ of the advective speed to the internal Rossby wave speed is a better suited nondimensional parameter when it is a question of distinguishing between the appearance of classical baroclinic modes or barotropic Rossby basin modes as the first oscillatory instabilities of the flow. Note also that $\Omega$ is the parameter that appears naturally in the definition of the geostrophic contours $\hat{\phi}$, the characteristics along which information propagates in the basin (Rhines and Young, 1982). By definition, $\hat{\phi}=y+\Omega \phi$, where $\phi=H_{1} / H \psi_{1}+H_{2} / H \psi_{2}$ is the barotropic streamfunction.

\section{b. Numerical methods}

Instead of relying on forward time integration to compute the different equilibrium states of the system for a different set of parameters, we have used continuation methods to solve directly for a branch of steady-state solutions, as a nondimensional parameter is varied. More details on the continuation methods and examples of their application to oceanographic problems can be found in Dijkstra (2005). The idea behind these methods is that by monitoring the linear stability of a branch of steady-state solutions, one can explain the onset of a complex time-dependent circulation through a succession of bifurcations. For the case of a 2-layer QG model, the generally complex eigenfunctions $\hat{\psi}_{n}(x, y)=$ $\hat{\psi}_{n, r}+i \hat{\psi}_{n, i}$ (one for each density layer) and the corresponding eigenvalue $\sigma=\sigma_{r}+i \sigma_{i}$ obtained from solving the linear stability problem for a given equilibrium flow provide the spatial structure, period $T=2 \pi / \sigma_{i}$ and growth rate $\sigma_{r}$ of a specific perturbation $\psi_{n}^{\prime}(x, y, t)$, or internal mode, that destabilizes the flow,

$$
\psi_{n}^{\prime}(x, y, t)=\operatorname{Real}\left(\hat{\psi}_{n} e^{\sigma t}\right)=e^{\sigma_{r} t}\left[\hat{\psi}_{n, r}(x, y) \cos \sigma_{i} t-\hat{\psi}_{n, i}(x, y) \sin \sigma_{i} t\right] .
$$

The bifurcation points are the critical values of the control parameter (usually, the Reynolds number), where the real part of one or more eigenvalues changes sign, implying modification of the stability properties of the flow. At saddle-node and pitchfork bifurcations, a single real eigenvalue crosses the imaginary axis, which is linked to the appearance of multiple steady states. At a Hopf bifurcation, a pair of complex conjugated eigenvalues crosses the imaginary axis, which is linked to the destabilization of the flow to an oscillatory mode.

Previous studies have shown that only a small number of real and complex eigenvalues are involved in the transition of the double-gyre ocean circulation from steady equilibrium 
Table 1. Values of the dimensional and nondimensional parameters used in the wind-driven and thermally-driven calculations. Two different values for the internal deformation radius $R_{d}$ are considered, corresponding to $\Omega=1.2$ or $\Omega=0.3$. The parameters which are forcing specific, $U_{w}, U_{T}, \delta_{T}$, are provided in the text as the different calculations are presented.

$\begin{array}{lll} & L=1000 \mathrm{~km} & f_{0}=1 \times 10^{-4} \mathrm{~s}^{-1} \\ \text { Dimensional } & H_{1}=860 \mathrm{~m} & \beta_{0}=2 \times 10^{-11} \mathrm{~m}^{-1} \mathrm{~s}^{-1} \\ \text { parameters } & H_{2}=2140 \mathrm{~m} & \rho_{0}=1000 \mathrm{kgm}^{-3} \\ & U=0.02 \mathrm{~ms}^{-1} & R_{d}=29 \text { or } 58 \mathrm{~km} \\ & \nu=4000-200 \mathrm{~m}^{2} \mathrm{~s}^{-1} & \\ \text { Nondimensional } & \delta_{12}=0.4 & \beta=1000 \\ \text { parameters } & R e=5-100 & \Omega=1.2 \text { or } 0.3\end{array}$

to irregular behavior (Dijkstra, 2005). To determine these most unstable eigenvalues we have employed the same code as in Dijkstra and Katsman (1997), but now using the JacobiDavidson QZ method (Sleijpen and Van Der Vorst, 1996). The equations are solved on a $65 \times 65$ non-equidistant grid with the same stretching parameters as in Dijkstra and Katsman (1997). The stretched grid is advantageous since it provides finer resolution only in the parts of the basin (the western boundary layer and the mid-latitude jet), where strong solution gradients are expected. More details on the numerical methods are provided in Dijkstra (2005).

\section{Results}

We will present next the basic bifurcation diagrams for a thermally-only and windonly forced double-gyre circulation with the Reynolds number $R e$ in the role of a control parameter. The values of the common parameters used in the calculations are listed in Table 1. The parameters which are forcing specific, i.e. $U_{w}, U_{T}, \delta_{T}$, will be defined as the different calculations are presented. We are using two different values $\Omega=1.2$ and $\Omega=0.3$ for the ratio of the advective speed to the long baroclinic Rossby wave speed. Assuming that the forcing and thus the horizontal velocity scale $U$ are kept unchanged, these two values for $\Omega$ can be thought of as the case of an ocean basin with respectively small/large internal deformation radius $\left(R_{d} \approx 29 \mathrm{~km}\right.$ for $\Omega=1.2$ vis $R_{d} \approx 58 \mathrm{~km}$ for $\Omega=0.3$ ), where the internal Rossby waves are slower/faster than the background flow.

\section{a. Wind-forced case}

In order to provide continuity with previous studies, we are presenting first the case of a wind-only driven double-gyre circulation with zero cross-isopycnal flux $w_{*}$, i.e. $U_{T}=0$, $\delta_{T}=\infty$. The applied wind stress has a magnitude $\tau_{0}=0.34 \mathrm{~N} / \mathrm{m}^{2}$ with corresponding advective velocity scale $U=2 \mathrm{~cm} / \mathrm{s}$, resulting in a $17 \mathrm{~Sv}$ Sverdrup transport for the upper layer and an inertial boundary layer thickness $\sqrt{U / \beta_{0}} \approx 32 \mathrm{~km}$. In terms of the 
nondimensional parameters, this choice of values implies $U_{w}=\beta=1000$ (from Eqs. (2a) and (2b) and using the values in Table 1).

When describing a branch of steady-state solutions as function of the Reynolds number, we use as norm of the solution the maximum of the (nondimensional) baroclinic streamfunction, $\tau=\psi_{1}-\psi_{2}$, indicating the intensity of the subtropical gyre. In Figures $2 \mathrm{a}$ and $2 \mathrm{~b}$, the branches of steady-state solutions emanating from the linear Munk solution for a viscous wind-driven double-gyre, are plotted for the two cases $\Omega=1.2$ and $\Omega=0.3$, respectively. On these branches, the circulation consists of a double-gyre flow that is anti-symmetric with respect to the basin mid-latitude and becomes progressively more inertially dominated as the Reynolds number is increased. This translates into strengthening of the subtropical gyre and growing values for max $\tau$ as a function of the Reynolds number. Looking back at Eqs. (2a) and (2b), one can see that the only way in which $\Omega$ can influence the steady regime ocean dynamics is through the advection of stretching vorticity, $F_{n} J\left(\psi_{n}, \psi_{1}-\psi_{2}\right)$. The cross-isopycnal flux, $F_{n} w_{*}$ does not depend on $\Omega$, independent of whether thermal forcing is included or not. Because for the steady wind-driven circulation the motion is confined to the upper layer only, the advection of stretching vorticity is identically zero. Consequently, the branches of steady-state solutions for $\Omega=1.2$ and $\Omega=0.3$ in Figures $2 \mathrm{a}$ and $2 \mathrm{~b}$ are identical.

Although the steady-state wind-driven solutions are independent of $\Omega$, their linear stability is not. The most obvious difference is that the threshold for linear stability of the flow, as determined by the Reynolds number where the first bifurcation occurs, depends on $\Omega$. One can see in Figures $2 \mathrm{a}$ and $2 \mathrm{~b}$ that for $\Omega=1.2$ the circulation loses its linear stability at $R e_{H_{1}}=19.3$, while for $\Omega=0.3$, the critical threshold is $R e_{P_{1}}=30.4$. This is consistent with the idea that the larger the value of $\Omega$, the smaller is the internal deformation radius and the lower is the threshold for instability of the flow.

In addition to that, although in both configurations saddle-node, pitchfork and Hopf bifurcations are present, the order of their occurrence is different. In Figures $2 a$ and $2 b$ the locations of only the first pitchfork (marked $P_{1}$ ), the first saddle-node (marked $L_{1}$ ) and the first two Hopf bifurcations (marked $H_{1}$ and $H_{2}$ ), are shown. While the positions of the saddle-node and pitchfork bifurcations are almost unchanged between the two cases $\Omega=1.2$ and $\Omega=0.3$, the Hopf bifurcations shift to a larger Reynolds number for the case $\Omega=0.3$. Consequently, for the ocean basin with smaller deformation radius (larger $\Omega$ ), the pitchfork bifurcation is preceded by Hopf bifurcations. This signifies that the antisymmetric double-gyre circulation first becomes unstable to oscillatory modes, before the emergence of multiple equilibria through the symmetry breaking pitchfork bifurcation. More specifically at $P_{1}$, branches of asymmetric steady-state solutions appear on which the double-gyre circulation is characterized with either the subtropical or subpolar gyre being of larger intensity and spatial extent. These asymmetric solution branches are not plotted since they are not the subject of our study here, but their role for the internal wind-driven circulation variability is well documented (Cessi and Ierley, 1995; Simonnet and Dijkstra, 2002; Primeau, 2002). For the ocean basin with larger deformation radius (smaller $\Omega$ ), the 
a)

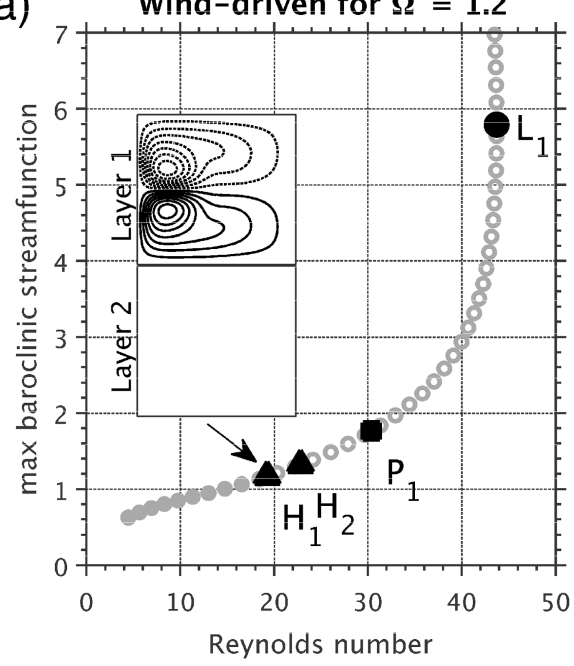

c)

$\mathbf{H}_{\mathbf{1}}$ :

(113.5 days)
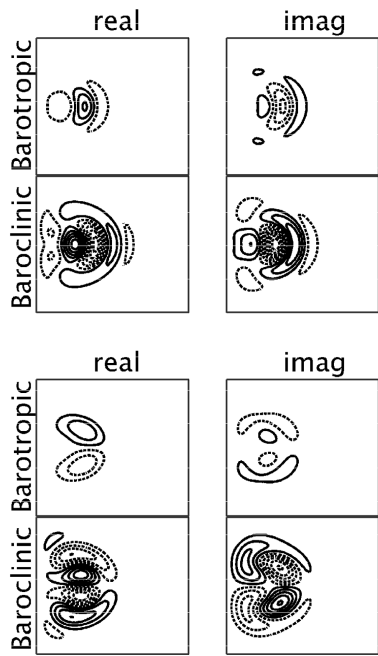

b)

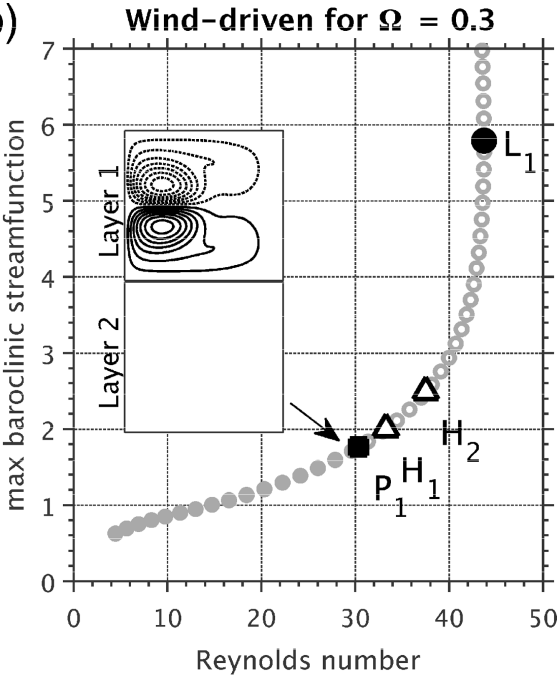

d)
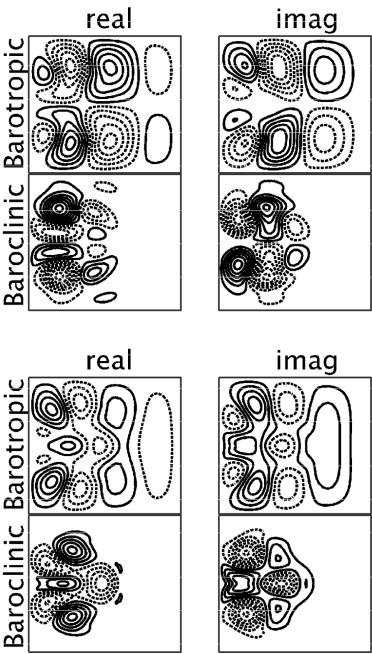

Figure 2. Bifurcation diagram for a wind-driven double-gyre circulation with a) $\Omega=1.2$ and b) $\Omega=0.3$. Filled (empty) circles denote linearly stable (unstable) steady-state solutions. H, P and L stand for Hopf, pitchfork and saddle-node bifurcation point, respectively. The steady-state solution at the critical Reynolds number is shown in the insert (contour interval 0.1). The spatial structure (by vertical modes) and periods (in days) for the first two oscillatory modes $H_{1}$ and $H_{2}$ are given in c) for $\Omega=1.2$ and in d) for $\Omega=0.3$. Solid (dashed) lines denote clockwise (anticlockwise) motion. 
situation is exactly the opposite - the pitchfork bifurcation, or the appearance of multiple equilibria, occurs before the Hopf bifurcations, and hence before the destabilization to oscillatory modes.

Another, more subtle difference, but which is the feature to which we would like to bring attention with the present study, is that depending on $\Omega$ the first Hopf bifurcations correspond to different types of oscillatory modes. For $\Omega=1.2$ (small deformation radius), the oscillatory modes destabilizing the flow are of the classical baroclinic type, as described first in Dijkstra and Katsman (1997). They have inter-monthly time periods, a phase difference between the streamfunction perturbations in the two layers, and spatial amplitudes confined to the region of the basin mid-latitude jet and recirculation gyres. In Figure $2 c$ the barotropic and baroclinic part of the eigenvectors, which eigenvalues cross the imaginary axis at the bifurcation points $H_{1}$ and $H_{2}$, are plotted for the case $\Omega=1.2$. Note that the solution to the linear stability problem determines the eigenvectors only up to a constant factor. Thus we have chosen to use the maximum value of the real upper layer eigenvector as a normalization, which is equivalent to setting the maximum amplitude of the upper layer perturbation streamfunction at time $t=0$ to one (see Eq. (5)). It can be seen that the $H_{1}$ baroclinic mode is symmetric with respect to the basin mid-latitude and represents meandering of the jet with period 113.5 days. The $\mathrm{H}_{2}$ baroclinic mode on the other hand is anti-symmetric with respect to the basin mid-latitude and represents oscillations of the recirculation gyres associated with weakening and strengthening of the mid-latitude jet with period 192.3 days. Both these modes are consistent with the baroclinic oscillatory modes previously described in the literature (Dijkstra, 2005).

For $\Omega=0.3$ (large deformation radius), the Hopf bifurcations $H_{1}$ and $H_{2}$ correspond instead to the destabilization of the flow to barotropic basin modes, as shown in Figure $2 \mathrm{~d}$. The period of the oscillations is shorter with a monthly timescale (50-54 days), while horizontally the modes consist of several counter-rotating cells, spread out over the entire basin. Their barotropic part closely resembles the spatial structure and phase propagation characteristics of the theoretical 1 by 2 and 2 by 1 barotropic Rossby basin modes respectively, while their baroclinic part represents simply the advection of the interface displacement by that basin mode. As a reminder, the barotropic Rossby basin modes constitute free modes of oscillation of an ocean at rest, with analytical solutions known for the case of a flat-bottom square basin ocean (Pedlosky, 1987). The internal modes $H_{1}$ and $H_{2}$, being the eigenvectors of a non-zero equilibrium solution, are thus not pure basin modes but represent instead basin modes that are modified by the background circulation. There are some departures from the exclusively westward theoretical phase propagation and distortion in the spatial pattern of the modes due to the interaction with the background flow, especially in the region of the recirculations. Also, the period of the theoretical barotropic $n$ by $m$ basin mode in a square basin at rest is given by $T_{n, m}=4 \pi^{2} \sqrt{n^{2}+m^{2}} /\left(L \beta_{0}\right)$ (Pedlosky, 1987), which leads for $L=1000 \mathrm{~km}$ and $\beta=2 \times 10^{-11} \mathrm{~m}^{-1} \mathrm{~s}^{-1}$ to $T_{1,2}=T_{2,1}=51$ days. This is close, but not exactly equal to the period of the oscillatory modes $H_{1}$ and $H_{2}$ found here, $T_{H_{1}}=53.4$ days and $T_{H_{2}}=50.0$ days. 
It is known that the barotropic Rossby basin modes are part of the spectrum of the closed basin double-gyre circulation. They have been reported as first Hopf bifurcations mainly in barotropic and reduced gravity models (Dijkstra and Katsman, 1997). In multi-layer model studies, where bifurcation analysis is used to explore the origin of the internal low-frequency ocean variability, attention is paid mostly to the lower frequencies classical baroclinic and gyre modes, while focus is rarely put on the basin modes which are higher frequency oscillations. We argue also in the next section of this paper that in order to observe the barotropic basin modes as the primary oscillatory instability of the double-gyre flow, the system has to be in a specific parameter regime.

\section{b. Thermally-forced case}

As a complement to the more widely (if not exclusively) studied wind-driven case, we will present in this section the basic bifurcation diagram for a thermally-only driven ocean. Before going into details about the stability of the flow, it is instructive to examine first the properties of the thermally-driven circulation in the linear limit. This will provide us with a reference circulation and a justification for why the study of a thermally-only driven ocean may be of value.

$i$. The linear thermally-driven circulation. For a sine thermal forcing (which implies no net heating/cooling) and a closed basin, the resulting thermally-only forced circulation is again a double-gyre flow (Pedlosky and Spall, 2005). Unlike the wind-driven case however, the circulation extends to both layers. This is due to the fact that the wind stress curl acts only on the top layer, while the cross-isopycnal flux acts as a forcing for both layers. If the governing Eqs. (2a) and (2b) are rewritten by vertical modes, where $\phi=\psi_{1} H_{1} / H+\psi_{2} H_{2} / H$ and $\tau=\psi_{1}-\psi_{2}$ are respectively the barotropic and baroclinic streamfunctions, then in the steady, linear, inviscid limit we have that

$$
\begin{aligned}
& \beta \frac{\partial \phi}{\partial x}=\frac{H_{1}}{H} U_{w} S_{w}(y), \\
& \beta \frac{\partial \tau}{\partial x}=U_{w} S_{w}(y)+\frac{\beta}{\delta_{T}}\left(\tau+U_{T} S_{T}(y)\right) .
\end{aligned}
$$

Thus, in the absence of wind forcing the linear circulation is purely baroclinic. A vertically integrated thermally-driven background flow can be generated only through the nonlinear advection terms, that couple the barotropic and baroclinic vertical modes. These terms are absent in the linearized Eqs. (6a) and (6b), but it is straightforward to see that the Jacobian terms from the original Eqs. (2a) and (2b) will lead to coupling of the barotropic and baroclinic vertical modes. This means that in the absence of wind forcing, the barotropic flow is weak, proportional to $\delta_{I}^{2}$ (or $1 / \beta$ ), with its magnitude completely vanishing in the linear limit.

From a basin-scale circulation point of view, we know that a strong enough barotropic circulation can arrest the westward Rossby wave propagation and create regions of closed 
geostrophic contours isolated from the eastern boundary, where the circulation departs from the simple Sverdrup balance (Rhines and Young, 1982). Although a thermally-only driven flow is without doubt not representative for the real ocean circulation, it provides an instructive hypothetical setting, given that only a weak vertically integrated circulation is generated, to explore the role of the linear limit circulation structure and its associated flow of information for the onset of time-dependence in the system.

ii. Bifurcation diagram for a thermally-driven circulation. For the thermally-only forced calculations we have limited the parameter space to explore by choosing a fixed thermal scale, $\delta_{T}=1$. This choice signifies that the timescale $\gamma$, on which the interface displacement is relaxed toward the prescribed equilibrium profile, matches the travel time across the basin $T_{R}=L / \beta_{0} R_{d}^{2}$ for the long internal Rossby waves, where $T_{R}=2$ years $\left(T_{R}=0.5\right.$ years $)$ for $\Omega=1.2(\Omega=0.3)$, respectively. As a result, the thermal relaxation has only a weak effect on the internal Rossby wave propagation, since it does not inhibit their propagation across the basin. The opposite limit, not considered within this paper, would be to use a relaxation timescale $\gamma$ much shorter than $T_{R}$. In this case, the Rossby waves would be strongly affected by the interface relaxation and remain trapped near the eastern boundary, while the interface displacement in the basin interior would closely match the specified target profile $h_{0} S_{T}(y)$. Concerning the amplitude of the equilibrium profile that sets up the advective velocity scale for the thermally-only forced circulation, we have chosen $h_{0}$ so that the upper layer circulation is characterized, in the linear limit, with the same order interior velocity and transport as the wind-only driven case. This leads from Eq. (6b) after some algebra to $U_{T}=2$ and $h_{0}=294 \mathrm{~m}\left(h_{0}=74 \mathrm{~m}\right)$ for $\Omega=1.2(\Omega=0.3)$, respectively ${ }^{4}$.

The branches of thermally-forced, steady-state solutions described by the maximum baroclinic streamfunction as a function of the Reynolds number, are plotted in Figures 3a and $3 \mathrm{~b}$ for the two cases $\Omega=1.2$ and $\Omega=0.3$, respectively. The branches originate in the viscous, weakly nonlinear limit from equilibria with approximately the same norm, consistent with the linearized baroclinic mode Eq. (6b), that is independent of $\Omega$. However, unlike for the wind-only driven case, the branches for the two values of $\Omega$ progressively diverge, when followed to larger Reynolds numbers. This is due to the fact that the thermal forcing, unlike the wind stress curl, sets in motion both layers and as a result the stretching vorticity and value of $\Omega$ matter for the steady-regime dynamics.

As the flow becomes more nonlinear, the intensity of the subtropical and subpolar gyres increases, although less dramatically than for the wind-driven circulation. The reason for this is the relaxation thermal forcing that acts to limit the interface deviations by restoring $\eta$ to the prescribed equilibrium profile. It can be seen from the marginally-stable steady-state solutions included in Figures $3 a$ and $3 b$, that the gyres increase in intensity because of the

4. From Eq. (6b), the linear thermally-only forced solution for the baroclinic streamfunction is $\tau(x, y)=$ $U_{T} S_{T}(y)\left(e^{\frac{x-1}{\delta_{T}}}-1\right)$. The scale for the upper layer streamfunction is then $\psi_{1} \sim \frac{H_{2}}{H} \tau \sim \frac{H_{2}}{H} U_{T}\left(1-e^{-\frac{1}{\delta_{T}}}\right)$, which we want to be equal to one (the value for the wind-driven case). 
a) Thermally-driven for $\Omega=1.2$

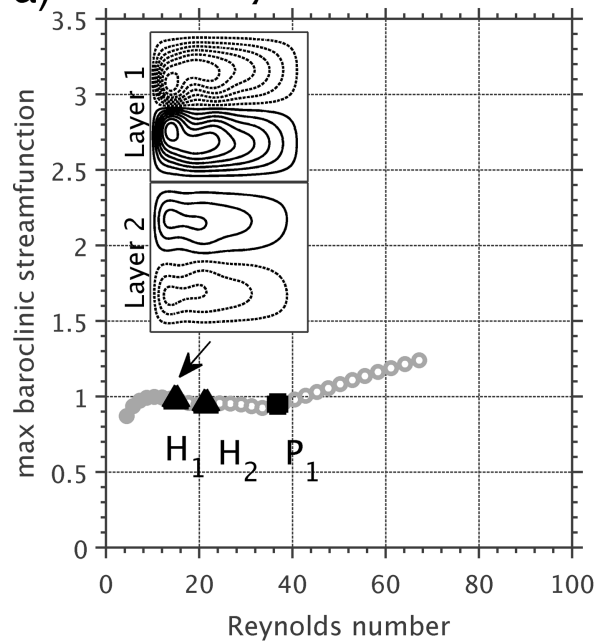

C)
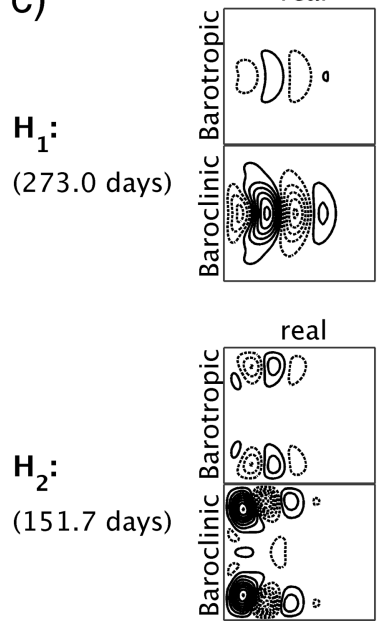

b) Thermally-driven for $\mathbf{\Omega}=\mathbf{0 . 3}$

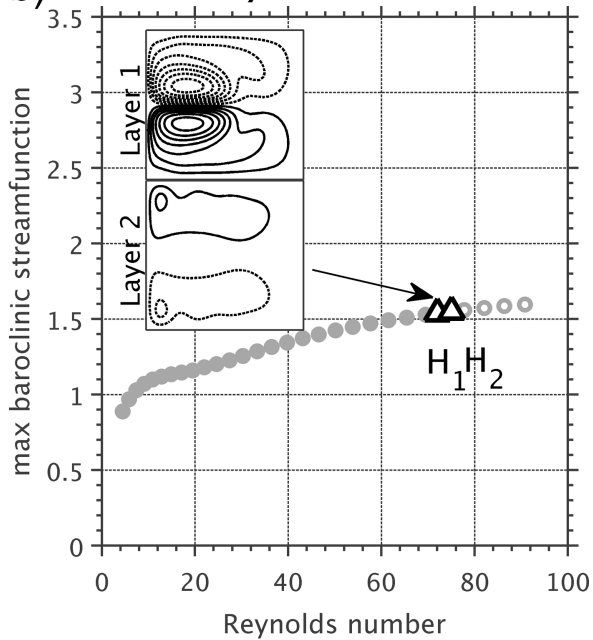

d)
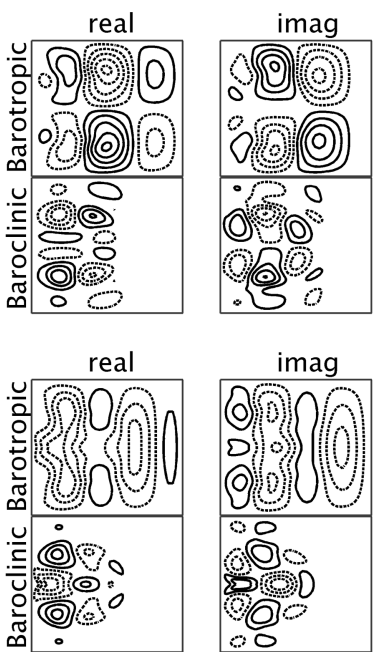

Figure 3. Bifurcation diagram for a thermally-driven double-gyre circulation with a) $\Omega=1.2$ and b) $\Omega=0.3$. The steady-state solution at the critical Reynolds number is shown in the insert (contour interval 0.1). The spatial structure (by vertical modes) and periods (in days) for the first two oscillatory modes $H_{1}$ and $H_{2}$ are given in c) for $\Omega=1.2$ and in d) for $\Omega=0.3$.

formation of recirculations that have both a barotropic and a baroclinic component. This is an illustration that, although the thermal forcing does not directly drive a barotropic flow, a steady barotropic circulation is generated because of the nonlinear dynamics.

Although the thermally-driven, steady-state solutions differ in several ways from the wind-driven ones, we can make qualitatively similar observations concerning the dependence of the oscillatory modes on $\Omega$, the ratio of the flow advective speed to the long internal 
Rossby wave speed. For the smaller $\Omega$ (larger deformation radius) thermally-forced doublegyre flow, the first two Hopf bifurcations correspond to barotropic basin modes (Fig. 3d). The first Hopf bifurcation at $R e_{H_{1}}=72.1$ has the structure of a 1 by 2 basin mode with period $T_{H_{1}}=54.0$ days. The second Hopf bifurcation at $R e_{H_{2}}=75.1$ corresponds to a 2 by 1 basin mode with period $T_{H_{2}}=53.6$ days. Both internal modes are nearly identical to those for the wind-driven case. A difference from the wind-driven case is that we did not locate any symmetry-breaking pitchfork bifurcation below $R e=100$.

The larger $\Omega$ (smaller deformation radius) thermally-forced double-gyre flow has a lower Reynolds number threshold for linear instability. In addition, the first two Hopf bifurcations correspond instead to baroclinic modes (Fig. 3c). The first Hopf bifurcation at $R e_{H_{1}}=15.0$ is characterized with a spatial structure that is symmetric with respect to the basin midlatitude and leads to meandering of the mid-latitude jet with an inter-monthly period of $T_{H_{1}}=273.0$ days. In that sense, it is similar to the first oscillatory mode for the winddriven case. The second Hopf bifurcation for the thermally-forced case is actually two Hopf bifurcations. A second pair of complex eigenvectors (not shown) with a very similar spatial structure and imaginary part eigenvalue (i.e., same period) becomes unstable at approximately the same Reynolds number, $R e_{H_{2}}=21.4$, as the pair shown in Figures $3 \mathrm{a}$ and $3 \mathrm{c}$. These oscillatory modes are boundary intensified with the maximum amplitude occurring near the southern and northern walls of the domain. They lead to meandering of the zonal boundary layers with an inter-monthly period $T_{H_{2}}=151.7$ days. No such oscillatory modes have been reported for the wind-driven double-gyre in the literature. Based on energetics, which we will present in more detail in the next section, these new internal modes can be classified as baroclinic ones as well. Therefore, similar to the wind-driven case, the larger $\Omega$ (smaller deformation radius) thermally-forced circulation is characterized with baroclinic oscillatory modes with inter-monthly periods. Also similar to the wind-driven case, a symmetry breaking pitchfork bifurcation occurs at $R e_{P_{1}}=36.8$, when the flow is already linearly unstable to several oscillatory modes.

Another particularity of the bifurcation diagram of the thermally-forced double-gyre is that we did not detect any saddle-node bifurcations, at least within the range of Reynolds numbers covered here. For the wind-driven problem, the two (there is one farther down the branch) saddle-node bifurcations $\mathrm{L}$ on the anti-symmetric solution branch are associated with the approach to an inertial regime (Dijkstra and Katsman, 1997). Apparently, the transition to this regime in the thermally-driven case is more direct (without an unstable branch) and this can only occur when the two saddle-nodes have merged in a cusp. The physics of this merging are difficult to determine without further analysis.

\section{Barotropic basin modes versus baroclinic modes}

Examination of the bifurcation diagrams for the wind-only and thermally-only driven double-gyre flow revealed that despite differences in the steady-state solutions, a similar dependence on the ratio $\Omega$ of the flow advective speed to the speed of long internal Rossby 
waves exists, as to whether the first Hopf bifurcations correspond to baroclinic modes or barotropic basin modes. It can be shown furthermore that the first oscillatory internal modes differ not only in their spatial and temporal structure, but also in the instability process at their origin. In order to illustrate this, we will examine next the total perturbation energy budget as a function of $\Omega$ for the first two Hopf bifurcations in the wind-driven and the thermally-driven case.

The perturbation energy budget can be derived from the governing Eqs. (2) by decomposing the streamfunction into basic state $\Psi_{n}$ (with corresponding velocity field $U_{n}=$ $-\partial \Psi_{n} / \partial y$ and $\left.V_{n}=\partial \Psi_{n} / \partial x\right)$, which is the steady-state solution at a given Reynolds number, and a perturbation streamfunction $\psi_{n}^{\prime}$, which is the oscillatory mode of interest at this Reynolds number as determined from the linear stability analysis. Neglecting all nonlinear in $\psi_{n}^{\prime}$ terms, an equation for the evolution of the total perturbation energy $E^{\prime}=1 / 2 \iint \frac{H_{1}}{H} \overline{\left(\nabla \psi_{1}^{\prime}\right)^{2}}+\frac{H_{2}}{H} \overline{\left(\nabla \psi_{2}^{\prime}\right)^{2}}+\beta \Omega \frac{H_{1} H_{2}}{H^{2}} \overline{\left(\psi_{1}^{\prime}-\psi_{2}^{\prime}\right)^{2}}$ can be derived following, for example, Pedlosky (1987)

$$
\begin{aligned}
& \frac{\partial E^{\prime}}{\partial t}=\underbrace{\iint \sum_{n=1}^{2} \frac{H_{n}}{H}\left[\overline{\left(\frac{\partial \psi_{n}^{\prime}}{\partial x}\right)^{2}} \frac{\partial U_{n}}{\partial x}+\overline{\frac{\partial \psi_{n}^{\prime}}{\partial x} \frac{\partial \psi_{n}^{\prime}}{\partial y}}\left(\frac{\partial V_{n}}{\partial x}+\frac{\partial U_{n}}{\partial y}\right)+\overline{\left(\frac{\partial \psi_{n}^{\prime}}{\partial y}\right)^{2}} \frac{\partial V_{n}}{\partial y}\right]} \\
& \text { BT } \\
& +\underbrace{\iint \beta \Omega \frac{H_{1} H_{2}}{H^{2}}\left[\overline{\psi_{1}^{\prime} \frac{\partial \psi_{2}^{\prime}}{\partial x}}\left(U_{1}-U_{2}\right)+\overline{\left.\psi_{1}^{\prime} \frac{\partial \psi_{2}^{\prime}}{\partial y}\left(V_{1}-V_{2}\right)\right]}\right.}_{B C} \\
& -\underbrace{\iint \sum_{n=1}^{2} \frac{1}{R e} \frac{H_{n}}{H} \overline{\psi_{n}^{\prime} \nabla^{4} \psi_{n}^{\prime}}}_{\text {DISS }}-\underbrace{\iint \frac{H_{1} H_{2}}{H^{2}} \frac{\beta}{\delta_{T}} \overline{\left(\psi_{1}^{\prime}-\psi_{2}^{\prime}\right)^{2}}}_{R E S T} .
\end{aligned}
$$

In the equation above, the double integrals are taken over the area of the basin, the summation is over the two density layers, while the overbar indicates time-averaging over the period of the oscillatory mode.

The energy balance states that the rate of change of the total perturbation energy is a combination of the decay due to viscous dissipation (DISS) and thermal relaxation (REST, if present), and growth due to the barotropic (BT) and/or baroclinic (BC) conversion terms through which energy can be extracted from the background flow, if the conditions are favorable. The barotropic conversion term is proportional to the horizontal shear of the background flow; and if positive, indicates perturbations growing on the kinetic energy of the basic state or barotropic instability. The baroclinic conversion term is proportional to the vertical shear of the background flow; and if positive, indicates perturbations growing on the potential energy of the basic state or baroclinic instability. No flux terms are present in the integrated energy budget (7) because of the no-normal flow conditions at the basin walls. 
a)

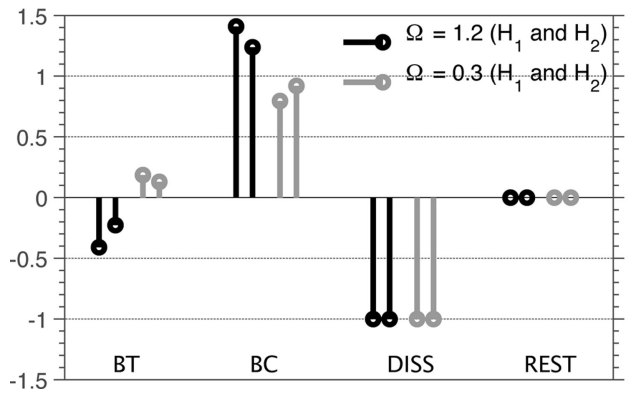

b)

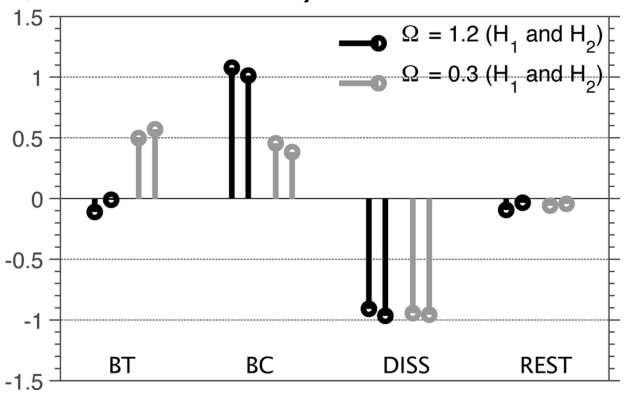

Figure 4. Perturbation energy budget for the first two oscillatory modes for a) the wind-driven doublegyre, and b) the thermally-driven double-gyre. For each value $\Omega=1.2$ and $\Omega=0.3$, the first bar corresponds to the first oscillatory mode $H_{1}$, while the second bar, to the second oscillatory mode $\mathrm{H}_{2}$. The four terms in the energy budget are: BT, barotropic energy conversion, BC, baroclinic energy conversion, DISS, viscous dissipation, and REST, damping due to interface restoring.

The perturbation energy budgets for the first two Hopf bifurcations for the wind-driven and the thermally-driven double-gyre and both values $\Omega=1.2$ and $\Omega=0.3$, are shown in Figures 4(a) and 4(b), respectively. In all cases, the energy budget is computed for a Reynolds number just above the corresponding bifurcation, so that the oscillatory mode of interest is unstable and slightly growing. In general, because the magnitude of the rate of change of $E^{\prime}$ depends on the choice of normalization for the eigenmode $\hat{\psi}_{n}$ as well as the growth rate $\sigma_{r}$, we have scaled all terms in the energy budget (7) by the sum of the absolute value of the two sink terms in the budget, DISS and REST. This does not affect the sign of the source terms BT and BC - the main information in which we are interested, while it makes the comparison between the different cases easier.

It can be seen in Figure 4 that for $\Omega=1.2$ in both the wind-driven and the thermallydriven case, it is the baroclinic conversion term that is positive and leads to overall growth of $E^{\prime}$, while the barotropic conversion term is negative. In other words, the first two oscillatory modes for $\Omega=1.2$ result from a baroclinic, or shear instability of the double-gyre flow. This is consistent with previous discussion of these modes in the literature (Dijkstra and Katsman, 1997) and is actually at the origin of their name. Note also, that the boundary intensified oscillatory modes for the thermally-driven double-gyre with $\Omega=1.2$ are indeed baroclinic modes as previously stated, since the sole source for their growth is the baroclinic conversion term. For $\Omega=0.3$ on the other hand, both the barotropic and the baroclinic conversion terms are positive and contribute to the overall growth of $E^{\prime}$, indicating that the basin modes result instead from a mixed barotropic-baroclinic instability. From the stability theory of zonal jets we know that the necessary condition for baroclinic instability of a zonal background flow with constant shear is that there is a change of the sign of the potential vorticity gradient between the upper and lower layer (Pedlosky, 1987). For an eastward flowing jet and the nondimensionalization applied here, this condition corresponds to $\Omega>H / H_{1} \approx 3.5$. In the case of the double-gyre flow, we seem to be able to get a positive 
a) $\Omega=U / \beta_{0} R_{d}^{2}=1.2$

Wind-driven

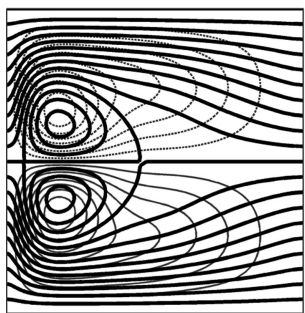

Thermally-driven

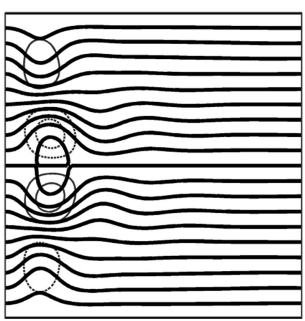

b) $\Omega=U / \beta_{0} R_{d}^{2}=0.3$

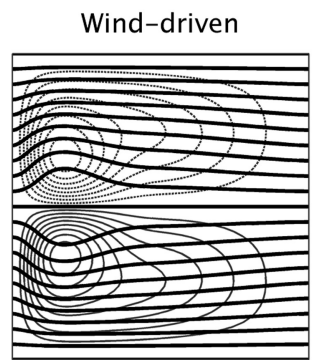

Figure 5. Barotropic streamfunction $\phi$ (in gray, contour interval 0.03) and geostrophic contours $\hat{\phi}=y+\Omega \phi$ (in black) for the wind-driven and the thermally-driven double-gyre with a) $\Omega=1.2$, and b) $\Omega=0.3$. The solution at $R e=12$ is shown, a Reynolds number below the linear instability threshold of the flow.

baroclinic energy conversion even below the baroclinic instability threshold for a zonal jet. This is not contradictory since the double-gyre flow contains non-zonal background flow that is always baroclinically unstable with no viscosity (Walker and Pedlosky, 2002; Spall, 2000).

The dependence on $\Omega$ of the type of oscillatory modes that destabilize the double-gyre circulation can be followed down the bifurcation tree below the threshold for linear instability. There is a range of Reynolds numbers below the critical one, where although the flow is linearly stable it still exhibits preference toward one or the other type of modes. In other words, if perturbed, the circulation would respond more strongly to perturbations that have the inter-monthly periods and spatial structure of the baroclinic modes in one case, and to those resembling the higher frequency barotropic basin modes in the other case. In order to investigate the reason for this predisposition of the double-gyre circulation toward different instability processes, we have examined the steady-state solutions at even lower Reynolds number, where the flow is only weakly nonlinear and stable.

In Figure 5, the barotropic part of the wind-driven and thermally-driven circulation for the two different values of $\Omega$ is plotted for $R e=12$, a small Reynolds number toward the beginning of the bifurcation diagrams, that is below the linear stability threshold of the flow in all cases (see Figs. 2 and 3). In this weakly nonlinear limit, the equilibrium solution, either wind-driven or thermally-driven, depends only slightly, if at all, on the value for $\Omega$. However, there is a fundamental difference in the shape of the geostrophic contours, $\hat{\phi}=y+\Omega \phi$. For $\Omega=1.2$, the circulation is fast enough to arrest the westward Rossby wave propagation and a region of closed geostrophic contours with non-Sverdrupian dynamics is evident in the western part of the basin. Incidentally, in this situation the preferred oscillatory modes of the flow when it eventually becomes unstable, are the baroclinic ones with maximum amplitude confined roughly to the closed geostrophic contour region. For $\Omega=0.3$ on the other hand, the flow is sluggish compared to the long Rossby wave speed and consequently does not affect the paths of Rossby wave propagation. Consequently, the geostrophic contours are 
nearly zonal and blocked on all latitudes. The preferred oscillatory modes of the flow in this case are the barotropic basin modes instead.

In order to test whether there is indeed a relationship between the shape of the geostrophic contours in the weakly nonlinear limit and the type of oscillatory modes to which the double-gyre eventually becomes unstable, we have expanded the bifurcation diagrams in the $\Omega / \operatorname{Re}$ parameter space. More specifically, we varied the ratio $\Omega$ that controls the shape of the geostrophic contours between 0.2 and 1.8, which corresponds to an internal Rossby deformation radius between $70 \mathrm{~km}$ and $23 \mathrm{~km}$ respectively, while all other parameters including the forcing, were kept unchanged and as specified in Table 1. The locations of the first four Hopf bifurcations (or less in some cases) and the first pitchfork bifurcation are shown in the $\Omega / R e$ parameter space for the wind-driven and the thermally-driven double-gyre in Figures $6 \mathrm{a}$ and $6 \mathrm{~b}$, respectively. The type of oscillatory mode corresponding to each Hopf bifurcation is rendered through the choice of symbol used - filled triangle for a baroclinic mode, and empty triangle for a basin mode. It can be seen that for both the wind-driven and the thermally-driven case the first Hopf bifurcations correspond to baroclinic modes for the large values of $\Omega$, while the basin modes start dominating for the small values of $\Omega$.

To further refine this analysis, using the linear limit solution for a wind-driven doublegyre, $\phi=\left(H_{1} / H\right)(1-x) \sin (2 \pi y)$, it is possible to derive a critical value for the ratio $\Omega$ above which closed geostrophic contours are present ${ }^{5}$. Characteristics isolated from the eastern wall will occur only if the meridional gradient of the geostrophic contours at midlatitude $\partial_{y} \hat{\phi}(x, y=1 / 2)=1+\Omega \partial_{y} \phi$ vanishes within the domain, i.e for $0<x_{0}<1$, leading to the following condition on $\Omega$

$$
x_{0}=1-\frac{1}{2 \pi \Omega \frac{H_{1}}{H}}>0 \Rightarrow \Omega>\frac{1}{2 \pi \frac{H_{1}}{H}} .
$$

For the set of parameters used here, this value is $\Omega_{c}=0.56$ and is drawn in Figure 6a with a dashed line. It can be seen that for the wind-driven double-gyre the switch from baroclinic modes to basin modes as the most unstable oscillatory modes of the system occurs approximatively at $\Omega_{c}$.

For the thermally-only forced case, since there is no barotropic circulation in the linear limit, we cannot determine an a priori critical value for the ratio $\Omega$ as with the wind-driven double-gyre, above which closed geostrophic contours are present. Furthermore, the shape of the geostrophic contours in this case depends not only on $\Omega$ but on the Reynolds number as well, given that the characteristics are computed solely with the nonlinearly-driven barotropic flow, as obtained from the numerical solution. Therefore, discussing the shape of the geostrophic contours of the thermally-driven double-gyre in the weakly nonlinear limit is a sensitive issue. Nevertheless, for comparison purposes and since we made the

5. If one considers instead a solution for $\phi$ including a Munk western boundary layer, it is again possible to derive a critical value for the ratio $\Omega$. The so found $\Omega_{c}$ is approximatively the same as when only the interior solution for $\phi$ is used, while the algebra involved is much more lengthy. 

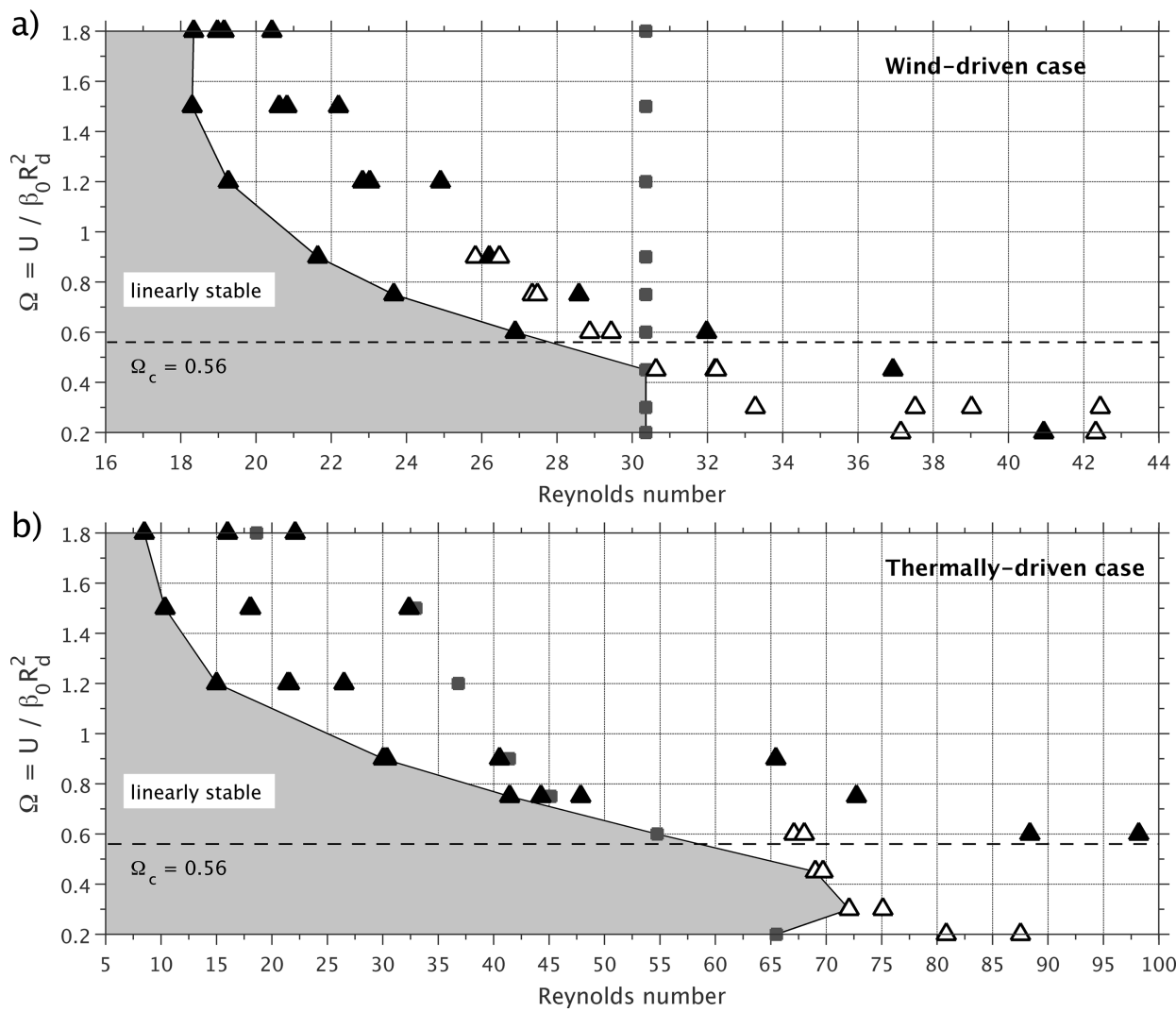

Figure 6. Bifurcation diagram in the $\Omega / R e$ parameter space for a) the wind-driven double-gyre, and b) the thermally-driven double-gyre. Shown are the first four Hopf bifurcations (triangle symbol) and the first pitchfork bifurcation (square symbol) below $R e=100$. Filled (empty) triangles indicate a Hopf bifurcation associated with a baroclinic oscillatory mode (barotropic basin mode). The dashed line at $\Omega_{c}=0.56$ marks the critical value above which closed geostrophic contours are present in a linear wind-driven double-gyre.

choice when setting up the calculations to match the interior linear velocities for the winddriven and the thermally-driven cases, we have added in Figure $6 \mathrm{~b}$ a reference line at the wind-driven critical value $\Omega_{c}=0.56$. It can be seen that a switch from barotropic basin modes to baroclinic modes as the most unstable oscillatory modes of the system occurs in the vicinity of $\Omega_{c}$ as well. There is a hint actually that the switch to baroclinic modes for the thermally-driven double-gyre happens at a slightly larger value than $\Omega_{c}$. This can be justified given that in the presence of thermal forcing only, for the same baroclinic flow magnitude, the barotropic part of the flow is weaker. Consequently, one would expect to find blocked characteristics and basin modes as the preferred oscillatory modes of the system over a wider range of Rossby wave speeds, i.e up to larger $\Omega$ values compared to the wind-driven case. 
Another observation that can be made from Figure 6 is that the threshold for linear instability of the double-gyre flow as determined by the occurrence of the first bifurcation, moves toward smaller Reynolds numbers as $\Omega$ is increased. Furthermore, a common feature of the wind-driven and the thermally-driven double-gyre is that the linear instability threshold is set by a symmetry-breaking pitchfork bifurcation for the small values of $\Omega$, and a Hopf bifurcation for large values of $\Omega$. The only cases that stand out are the thermally-driven $\Omega=0.3$ and $\Omega=0.45$ cases, where we did not locate any symmetry-breaking bifurcation below $R e=100$. Figure 6 also confirms our previous statement that for the wind-driven double-gyre the location of the pitchfork bifurcation does not change with $\Omega$, while for the thermally-driven double-gyre it moves toward smaller Reynolds numbers as $\Omega$ is increased. This can be rationalized given that a pitchfork bifurcation arises through a stationary mode associated with barotropic shear instability (Dijkstra, 2005). Since the horizontal shear of the background state seems to change much more strongly with $\Omega$ for the thermally-driven than for the wind-driven gyre, it is expected that the value of $\mathrm{P}$ will also shift more strongly in the former case.

As a final remark, we have tried here to put forward the argument that the ratio $\Omega$ of the flow advective speed to the long internal Rossby speed - the nondimensional parameter that controls the shape of the geostrophic contours, determines as well the type of the the most unstable oscillatory modes of the system. We have thus designed our numerical experiments so that we can explore the first Hopf bifurcations in the $\Omega / R e$ parameter space, while keeping all other nondimensional parameters of the system fixed. On the other hand, when analyzing the different regime of circulation in a basin, it is the Froude number $F=L^{2} / R_{d}^{2}=\beta \Omega$ that is more commonly used to characterize the system rather than $\Omega$ itself. Given that we have kept $\beta$ unchanged, one can argue than what we have described here is a dependence on $F$, not $\Omega$. However, experiments can be designed where we can vary simultaneously $F$ and $\beta$, while keeping their ratio $\Omega=F / \beta$ unchanged. In this case, although $F$ varies, the shape of the geostrophic contours in the weakly nonlinear limit remains unchanged since $\Omega=$ const and we would not expect to see any changes in the type of the most unstable oscillatory modes of the system. From the results of such experiments (not shown here), we have confirmed that it is indeed $\Omega$, not $F$, that matters as to whether the first Hopf bifurcations of the double-gyre system correspond to baroclinic modes or barotropic basin modes.

\section{Summary and discussion}

We have explored the onset of time-dependence in a simple 2-layer QG model of the double-gyre circulation with the emphasis put on how the type of oscillatory instabilities depends on the ratio $\Omega$ of the flow advective speed to the speed of internal long Rossby waves. This is the nondimensional parameter that controls the shape of the geostrophic contours in the linear limit and determines the extent of the Sverdrup balanced flow in the basin.

We considered two different types of forcing - the traditional wind-driven doublegyre and the less common, thermally-driven double-gyre, where cross-isopycnal velocity 
parameterized as restoring of the interface displacement to some equilibrium profile is included. The main difference between the wind-driven and the thermally-driven circulation lies within the vertical structure of the flow. In the absence of wind-forcing, the linear circulation is purely baroclinic. A thermally-driven barotropic flow can be generated only through the nonlinear advective terms that couple the barotropic and baroclinic vertical modes.

Because of the differences in the equilibrium solutions for the wind-driven and the thermally-driven double-gyre, there are predictably some differences in the stability properties of the flow. These differences concern mainly the overall linear stability threshold of the circulation, as well as the existence and critical Reynolds numbers for the bifurcations associated with non-oscillatory modes. However, we also uncovered a common, independent of the forcing, feature of the stability properties of the double-gyre flow. We found that on the branch of antisymmetric steady-state solutions, there is a relation between the nature of the leading oscillatory instabilities of the flow and the value of the ratio $\Omega$ of the flow advective speed to the speed of long internal Rossby waves. For large values of $\Omega$, when the flow is fast compared to the Rossby waves, the first Hopf bifurcations correspond to the classical baroclinic modes, with inter-monthly to annual time periods, maximum amplitude concentrated near the region of the mid-latitude jet and recirculation gyres, and arising from shear instabilities of the flow. For small values of $\Omega$, when the flow is slow compared to the Rossby waves, the first Hopf bifurcations correspond instead to barotropic basin modes, with shorter, monthly time periods, and arising from mixed barotropic-baroclinic instability of the flow. We found that the critical value for $\Omega$ that determines the threshold for the appearance of closed geostrophic contours in the linear limit circulation is a good guideline as to when the switch from barotropic basin modes to baroclinic modes as the leading oscillatory instabilities occurs. Although such a critical value cannot be derived strictly for the thermally-driven double-gyre, for which the barotropic part of the circulation vanishes in the linear limit, one can use as a reference an "equivalent" wind-driven flow that has the same interior baroclinic velocity.

It is a well established fact that both the barotropic basin modes and the classical baroclinic modes are part of the spectrum of the wind-driven double-gyre circulation. What our analysis shows is that a similar bifurcation diagram holds as well for the thermally-driven double-gyre, with the addition of some new, boundary intensified baroclinic modes, not previously reported in the wind-driven case. Our analysis also suggested that the important nondimensional parameter, that delimits the parameter space where the leading oscillatory instabilities are the barotropic basin modes from the parameter space where the leading oscillatory instabilities are the classical baroclinic modes instead, is the ratio $\Omega=U / \beta_{0} R_{d}^{2}$ of the flow advective speed to the speed of the long internal Rossby waves, and not for example the Froude number $F=L^{2} / R_{d}^{2}$.

For the purpose of this study we made the choice to analyze separately a wind-only and a thermally-only driven double-gyre, so that we can contrast them. More generally, a combined wind- and thermally-driven double-gyre will have stability properties a combination 
of the two. We expect a similar switch from barotropic basin modes to classical baroclinic modes as the leading oscillatory instabilities of the flow at approximatively the critical value for $\Omega$ where closed geostrophic contours appear in the weakly nonlinear limit. Concerning the multiple steady-state solution branches, we expect a pitchfork bifurcation with a critical Reynolds number depending on $\Omega$, and a lack of saddle-node bifurcation, similar to the thermally-driven double-gyre. These last two features are both associated with the presence of a cross-isopycnal velocity parameterized as interface relaxation, and consequently they will persist even if wind forcing is included as well.

The lack of saddle-node bifurcation in the presence of interface relaxation can have some important consequences for the low-frequency variability of the system. In previous bifurcation analyses of the double-gyre circulation, special attention has been paid to the gyre modes, which are low-frequency oscillatory modes with interannual to decadal time period, associated with weakening and strengthening of the mid-latitude jet. It has been shown that the origin of the gyre mode is related to the merging of two non-oscillatory modes, a P-mode and L-mode, associated with a pitchfork and saddle-node bifurcation, respectively (Simonnet and Dijkstra, 2002). If the L-mode is missing in a model including an interface relaxation parameterization of the cross-isopycnal flux, then that will imply that the gyre mode will be missing as well. Therefore, thermal relaxation may suppress the low-frequency variability of the system.

This finding about the dependence on $\Omega$ of the onset of time-dependent behavior in the system has some implications for the expected variability in ocean basins, depending on the relative size of the internal Rossby wave basin crossing timescale to the flow advective timescale. It suggests that high-frequency barotropic basin mode variability is more likely in small, low-latitude basins, while the baroclinic modes are to be prevailing in mid- and high-latitude basins and in large, Pacific/Atlantic Ocean size basins.

Acknowledgments. The authors would like to acknowledge comments from two anonymous reviewers that helped clarify the presentation. This work was partially completed during HGH graduate studies in the MIT/WHOI Joint Program in Physical Oceanography supported under NSF Grant OCE-0423975. HGH would like to thank also the Institute for Marine and Atmospheric Research Utrecht (IMAU), Netherlands for their hospitality and for providing the computer resources for this work. MAS was supported by NSF Grants OCE-042975 and OCE-0850416. This is JISAO contribution number 1828.

\section{REFERENCES}

Cessi, P. P. and G. R. Ierley. 1993. Nonlinear disturbances of western boundary currents. J. Phys. Oceanogr., 23, 1727-1735.

1995. Symmetry-breaking multiple equilibria in quasigeostrophic, wind-driven flows. J. Phys. Oceanogr., 25, 1196-1205.

Dijkstra, H. A. 2005. Nonlinear Physical Oceanography. A Dynamical Systems Approach to the Large Scale Ocean Circulation and El Niño. 2nd ed., Springer, 532 pp.

Dijkstra, H. A. and C. A. Katsman. 1997. Temporal variability of the wind-driven quasi-geostrophic double gyre ocean circulation: Basic bifurcation diagrams. Geophys. Astrophys. Fluid Dyn., 85, 195-232. 
Dijkstra, H. A. and M. J. Molemaker. 1999. Imperfections of the North-Atlantic wind-driven ocean circulation: continental geometry and wind stress shape. J. Mar. Res., 57, 1-28.

Held, I. M. 2000. The general circulation of the atmosphere, in 2000 Program in Geophysical Fluid Dynamics Proceedings, Woods Hole.

Jiang, S., F.-F. Jin, and M. Ghil. 1995. Multiple equilibria, periodic and aperiodic solutions in a wind-driven, double-gyre, shallow-water model. J. Phys. Oceanogr., 25, 764-786.

Nauw, J. J., H. A. Dijkstra, and E. Simonnet. 2004. Regimes of low-frequency variability in a threelayer quasi-geostrophic ocean model. J. Mar. Res., 62, 685-720.

Pedlosky, J. 1987. Geophysical Fluid Dynamics, 2nd ed., Springer, 728 pp.

Pedlosky, J. and M. A. Spall. 2005. Boundary intensification of vertical velocity in a beta-plane basin. J. Phys. Oceanogr., 35, 2487-2500.

Primeau, F. W. 2002. Multiple equilibria and low-frequency variability of the wind-driven ocean circulation. J. Phys. Oceanogr., 32, 2236-2256.

Rhines, P. B. and W. R. Young. 1982. A theory of the wind-driven circulation. I. Mid-ocean gyres. J. Mar. Res., 40(Suppl.), 559-596.

Schmeits, M. J. and H. A. Dijkstra. 2000. On the physics of the 9 months variability in the Gulf Stream region: combining data and dynamical systems analysis. J. Phys. Oceanogr., 30, 1967-1987.

Simonnet, E. and H. A. Dijkstra. 2002. Spontaneous generation of modes of low-frequency variability in the wind-driven ocean circulation. J. Phys. Oceanogr., 32, 1747-1762.

Sleijpen, G. L. G. and H. A. Van Der Vorst. 1996. A Jacobi-Davidson iteration method for linear eigenvalue problems. SIAM J. Matrix Anal. Appl., 17, 401-425.

Spall, M. A. 2000. Generation of strong mesoscale eddies by weak ocean gyres. J. Mar. Res., 58, 97-116.

Walker, A. and J. Pedlosky. 2002. Instability of meridional baroclinic currents. J. Phys. Oceanogr., $\underline{32,1075-1093 .}$

Received: 22 April, 2010; revised: 9 September, 2010. 